EGU2020-1325

https://doi.org/10.5194/egusphere-egu2020-1325

EGU General Assembly 2020

(c) Author(s) 2020. This work is distributed under

the Creative Commons Attribution 4.0 License.

\title{
Urban Thematic Exploitation Platform - supporting urban research with EO data processing, integrative data analysis and reporting
}

\author{
Felix Bachofer ${ }^{1}$, Thomas Esch ${ }^{1}$, Jakub Balhar ${ }^{2}$, Martin Boettcher ${ }^{3}$, Enguerran Boissier ${ }^{4}$, Mattia \\ Marconcini ${ }^{1}$, Annekatrin Metz-Marconcini ${ }^{1}$, Michal Opletal ${ }^{2}$, Fabrizio Pacini ${ }^{4}$, Tomas Soukup ${ }^{2}$, \\ Vaclav Svaton ${ }^{5}$, and Julian Zeidler ${ }^{1}$ \\ ${ }^{1}$ German Aerospace Center (DLR), German Remote Sensing Data Center (DFD), Wessling, Germany (felix.bachofer@dlr.de) \\ ${ }^{2}$ GISAT s.r.o., Prague, Czech Republic (tomas.soukup@gisat.cz) \\ ${ }^{3}$ Brockmann Consult GmbH, Hamburg, Germany (martin.boettcher@brockmann-consult.de) \\ ${ }^{4}$ Terradue Srl., Rome, Italy (fabrizio.pacini@terradue.com) \\ 5IT4Innovations, VSB-Technical University of Ostrava, Czech Republic (vaclav.svaton@vsb.cz)
}

Urbanization is among the most relevant global trends that affects climate, environment, as well as health and socio-economic development of a majority of the global population. As such, it poses a major challenge for the current urban population and the well-being of the next generation. To understand how to take advantage of opportunities and properly mitigate to the negative impacts of this change, we need precise and up-to-date information of the urban areas. The Urban Thematic Exploitation Platform (UrbanTEP) is a collaborative system, which focuses on the processing of earth observation (EO) data and delivering multi-source information on transsectoral urban challenges.

The U-TEP is developed to provide end-to-end and ready-to-use solutions for a broad spectrum of users (service providers, experts and non-experts) to extract unique information/ indicators required for urban management and sustainability. Key components of the system are an open, web-based portal connected to distributed high-level computing infrastructures and providing key functionalities for

i) high-performance data access and processing,

ii) modular and generic state-of-the art pre-processing, analysis, and visualization,

iii) customized development and sharing of algorithms, products and services, and

iv) networking and communication.

The service and product portfolio provides access to the archives of Copernicus and Landsat missions, Datacube technology, DIAS processing environments, as well as premium products like the World Settlement Footprint (WSF). External service providers, as well as researchers can make use of on-demand processing of new data products and the possibility of developing and deploying new processors. The onboarding of service providers, developers and researchers is 
supported by the Network of Resources program of the European Space Agency (ESA) and the OCRE initiative of the European Commission.

In order to provide end-to-end solutions, the VISAT tool on UrbanTEP allows analyzing and visualizing project-related geospatial content and to develop storylines to enhance the transport of research output to customers and stakeholders effectively. Multiple visualizations (scopes) are already predefined. One available scope exemplary illustrates the exploitation of the WSFEvolution dataset by analyzing the settlement and population development for South-East Asian countries from 1985 to 2015 in the context of the Sustainable Development Goal (SDG) 11.3.1 indicator. Other open scopes focus on urban green, functional urban areas, land-use and urban heat island modelling (e.g.). 